\title{
Incidence of myeloproliferative neoplasms in Calgary, Alberta, Canada
}

\author{
Jonathan Heppner ${ }^{1}$, Leonard Tu Nguyen², Maggie Guo ${ }^{2,3}$, Christopher Naugler ${ }^{2,3,4}$ \\ and Fariborz Rashid-Kolvear ${ }^{2,3,5^{*}}$
}

\begin{abstract}
Objective: The incidence of the combined myeloproliferative neoplasms (MPNs) was determined for a major Canadian city. Retrospective cases of MPN diagnoses (essential thrombocythemia, polycythemia vera, and primary myelofibrosis) between 2011 to 2015 were retrieved from the Southern Alberta Cancer Cytogenetics Laboratory's database at Alberta Public Laboratories.
\end{abstract}

Results: An incidence rate of 2.05 cases per 100,000 person-years (95\% Cl 1.73-2.41) was determined, giving an agestandardized Canadian incidence of 2.71 cases per 100,000 person years ( $95 \% \mathrm{Cl} 2.63-2.78)$. MPN diagnoses occurred at a wide age range of 8-93 (median 66) and an age-dependent increase in incidence. Incidence rates for the MPNs are first reported here for a Canadian population.

Keywords: Myeloproliferative neoplasm, Essential thrombocythemia, Polycythemia vera, Primary myelofibrosis, Cancer epidemiology, Hematological neoplasm

\section{Introduction}

The myeloproliferative neoplasms (MPNs) are clonal myeloid malignancies of the bone marrow classified by their unique genetic etiology and hematological histomorphologic features. The broadest classification is by BCR-ABL (Philadelphia chromosome) positive status for chronic myeloid leukemia (CML). The BCR-ABL negative MPNs, frequently characterized by mutations in Janus kinase 2 (JAK2), are further subclassified into essential thrombocythemia (ET), polycythemia vera (PV), primary myelofibrosis (PMF), and unclassifiable MPN [1]. Recently, mastocytosis was removed from classification as an MPN and while it has clinical and pathological features of an MPN, chronic myelomonocytic leukemia has been reclassified as a myelodysplastic syndrome/MPN disorder. Establishing clear discerning criteria between MPN classes requires summation of findings from peripheral blood slides, bone marrow morphology, cytogenetic and genetic alterations, and complete blood

\footnotetext{
*Correspondence: fariborz.kolvear@albertahealthservices.ca 2 Department of Pathology and Laboratory Medicine, Cumming School of Medicine, University of Calgary, Calgary, AB, Canada

Full list of author information is available at the end of the article
}

count $[1,2]$. Specific classification is essential not only for appropriate intervention strategies, but also prognostication. While myelodysplastic syndrome and acute myelogenous leukemia are not classified as MPNs, they are common transformations of the MPNs associated with significantly increased mortality, illustrating the need for appropriate diagnosis and prognosis.

Although the MPNs are rare cancers relative to solid tumors, they are an increasingly prevalent global healthcare issue and their epidemiology have been reported in the United States, Europe, and the Republic of Korea [3-7]. ET and PV consistently make up the majority of MPN cases in all populations studied with the mean age of diagnosis in the 50 to 60 range [4-7]. However, it has been noted that assessment of global incidence has been hampered by inconsistencies in diagnostic capacities and reporting between countries [3]. Furthermore, as diagnostic criteria change with increasing understanding of pathogenesis, so too will incidence and prevalence estimates. For example, it has been suggested that discovery of the JAK2 genetic mutation, along with improved testing diagnostic capacity, are factors potentially explaining increased prevalence and incidence over time [4]. There is also evidence of increased diagnostic stringency 
potentially decreasing perceived incidence [7], presumably through reduction of false positive results. Furthermore, MPN incidence is expected to rise in aging populations. Accordingly, monitoring the epidemiology of MPNs is essential to follow trends in population health, allocate appropriate healthcare resources, and evaluate the impact of ongoing diagnostic criteria refinement.

To date, there have been no reports on the incidence of the MPNs in the Canadian population. As a complement to the reported incidence of CML [8], we present the collective incidence of BCR-ABL-negative MPNs in Calgary, Alberta as well as their subclasses.

\section{Main text \\ Methods}

\section{Ethics approval}

This study was approved by the Health Research Ethics Board of the Alberta Cancer Committee (ID HREBA. CC-16-0830).

\section{Data source}

Patient data for this retrospective study was obtained from the Cancer Cytogenetics Laboratory at Alberta Public Laboratories (formerly Calgary Laboratory Services), which is the clinical testing facility serving the catchment area of over 1.8 million residents in Calgary and the surrounding Southern Alberta region. Blood and bone marrow were classified according to the $2008 \mathrm{WHO}$ guidelines via flow cytometry and hematopathologic examination [9]. Incident MPN cases were identified for the period of January 1, 2011 until December 31, 2015, and pathology reports were analyzed to determine MPN subclass as either ET, PV, PMF or unclassified MPN. ET is distinguished by sustained thrombocytosis (platelet count $\geq 450 \times 10^{9} / \mathrm{L}$ ), and proliferation and maturation of megakaryocytes with no known genetic or biological marker. PV is distinguished by elevated RBC production (measured by haemoglobin concentration, haematocrit, or RBC mass) with bone marrow biopsy showing hypercellularity with trilineage growth (erythroid, granulocytic, and megakaryocytic) and the JAK2 V617F mutation. PMF is distinguished by the proliferation of atypical megakaryocytes and granulocytes which may be accompanied by reticulin or collagen fibrosis in later development.

\section{Case identification}

Crude incidence rates were calculated for MPN cases in sex and 5-year age categories, and the age-standardized incidence for Canada was calculated using published methods [10]. Population estimates determined by postcensal coverage studies were retrieved from the Canadian Socio-Economic Information Management System (CANSIM) database from Statistics Canada [11, 12]. 95\% confidence intervals were calculated for each incidence rate by Wilson score interval for binomial proportions [13] using cumulative 2011-2015 population totals for Calgary and Canada, respectively.

\section{Results}

From 2011 to 2015, 139 new MPN diagnoses were made in the Calgary Metropolitan area, giving a crude incidence of 2.05 cases per 100,000 person-years (95\% CI 1.73-2.41, Table 1). There was slight gender bias with a male to female ratio of 1.14 . The range for age at diagnosis was broad from 8 to 93 with a median of 66 . Between the subclasses, ET had the highest incidence followed by PMF, PV, and unclassified MPN (MPN-U). The agestandardized incidence for Canada was 2.71 cases per 100,000 person-years (95\% CI 2.63-2.78).

The distribution of age- and sex-categorized MPN incidence rates is shown in Fig. 1. New cases were observed at early age in a few children, however there is a dramatic rise for patients older than 50 . The highest incidence was observed later in life past 75 years, particularly for males.

Table 1 Incidence rates and features of myeloproliferative neoplasms in Calgary (2011-2015)

\begin{tabular}{|c|c|c|c|c|c|}
\hline & ET & PV & PMF & MPN-U & All MPN \\
\hline New cases per year & 12.4 & 4.4 & 7.6 & 3.4 & 27.8 \\
\hline$M / F$ & 0.94 & 0.93 & 3.75 & 0.31 & 1.14 \\
\hline Median age of diagnosis & 61 & 67 & 71 & 57 & 66 \\
\hline Age range & $8-93$ & $13-88$ & $37-92$ & $14-92$ & $8-93$ \\
\hline Crude incidence per 100,000 person-years & 0.92 & 0.32 & 0.56 & 0.25 & 2.05 \\
\hline$(95 \% \mathrm{Cl})$ & $(0.71-1.16)$ & $(0.21-0.48)$ & $(0.40-0.76)$ & $(0.15-0.39)$ & $(1.73-2.41)$ \\
\hline $\begin{array}{l}\text { Age-standardized incidence for Canada per } \\
\text { 100,000 person-years }\end{array}$ & 1.16 & 0.44 & 0.80 & 0.31 & 2.71 \\
\hline$(95 \% \mathrm{Cl})$ & $(1.11-1.21)$ & $(0.41-0.47)$ & $(0.76-0.84)$ & $(0.28-0.33)$ & $(2.63-2.78)$ \\
\hline
\end{tabular}

ET, essential thrombocythemia; PV, polycythemia vera; PMF, primary myelofibrosis; MPN-U: myeloproliferative neoplasm-unknown; MPN, myeloproliferative neoplasm 


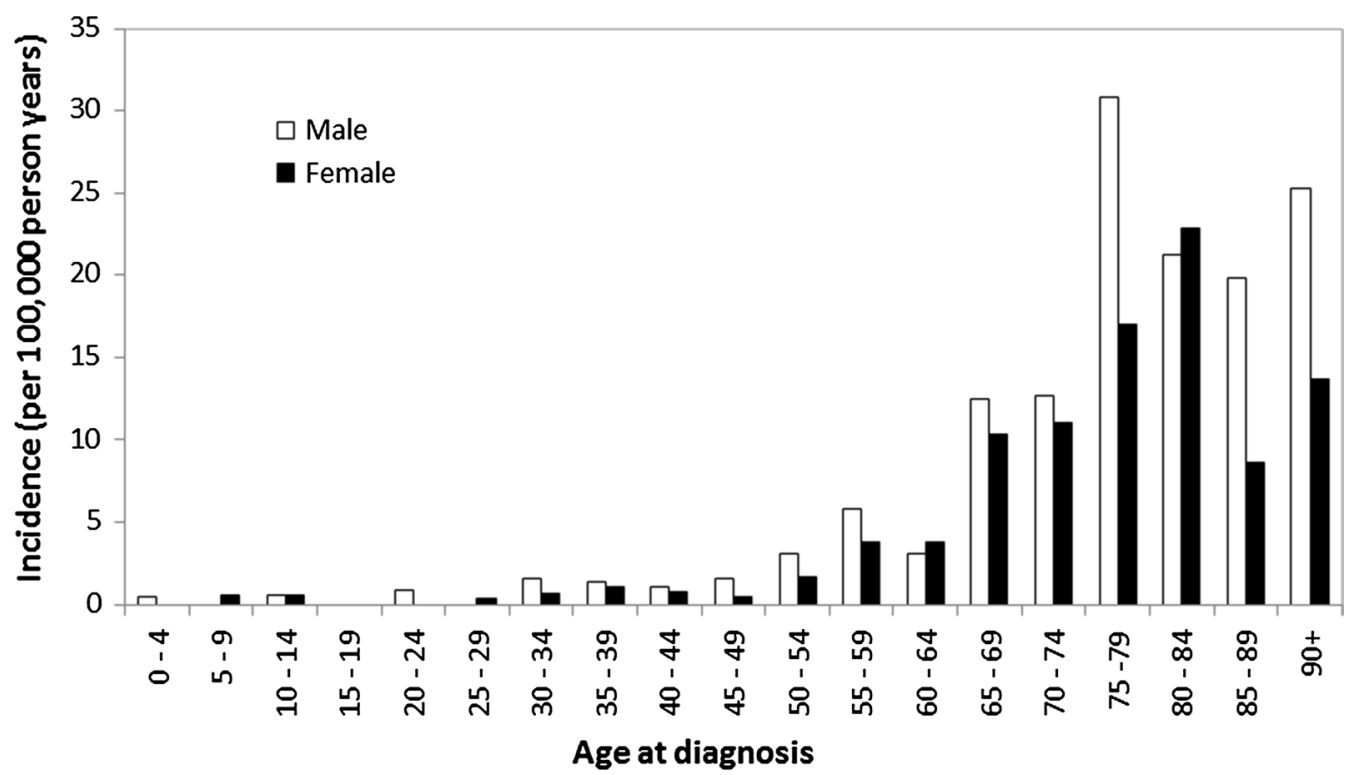

Fig. 1 Age and sex-categorized incidence rates of myeloproliferative neoplasms in the Calgary metropolitan area (2011-2015)

Table 2 Incidence rates (per 100,000 person-years) of MPNs

\begin{tabular}{|c|c|c|c|c|c|c|c|}
\hline \multirow[t]{2}{*}{ Region } & \multirow[t]{2}{*}{ Period } & \multicolumn{4}{|c|}{ Subclass } & \multirow[t]{2}{*}{ MPN } & \multirow[t]{2}{*}{ Source } \\
\hline & & ET & PV & PMF & MPN-U & & \\
\hline Canada & $2011-2015$ & 1.16 & 0.44 & 0.80 & 0.31 & 2.71 (non-CML), 3.58 (all) & Current study, [8] \\
\hline USA & 2008-2011 & 0.9 & 1.0 & 0.3 & NA & 4.2 (all) & SEER [4] \\
\hline Norway & 2010-2012 & 1.0 & 0.7 & 0.5 & 0.1 & 2.9 (non-CML) & {$[5]$} \\
\hline $\begin{array}{l}\text { Europe (18 coun- } \\
\text { tries) }\end{array}$ & 1995-2002 & 0.4 & 0.5 & 0.1 & NA & NA & {$[6]$} \\
\hline Korea & 2004-2013 & 2.4 & 1.2 & 0.4 & NA & NA & {$[7]$} \\
\hline
\end{tabular}

ET, essential thrombocythemia; PV, polycythemia vera; PMF, primary myelofibrosis; MPN-U, myeloproliferative neoplasm-unknown; NA, not available; SEER, surveillance, epidemiology, and end results registry

\section{Discussion}

The crude incidence reported here for myeloproliferative neoplasms in Calgary and standardized rate for Canada, which has a proportionally older population, are generally lower than corresponding values from other regions including the United States (Table 2). A direct comparison may not be straightforward due the changing diagnostic criteria of MPNs and population-specific factors such as age and genetic variance. Furthermore, incidence rates may be variable both between close populations as well as within populations over time $[4,6,7]$. Changing populations and refinements in diagnostic sensitivity, criteria, and reporting are thought to contribute to the increasing MPN incidence rate over the past decades.

The slight bias of MPNs for males over females is in agreement with the literature [3], however there is some variance between subtypes. There was almost four times as many male cases of PMF than female and the gender preference is flipped for the other subclasses. In literature values, the high proportion of male PMF incidence was also observed but not as strongly as observed here. Female preference in ET cases has also been remarked, however male incidence has been generally found to be more dominant for PV $[4,6,7]$. With a median age of diagnosis of 71, PMF incidence was generally older than the other subclasses. Age bias in the MPNs has been discussed; for example a recent report of ET had the highest incidence compared to other MPNs in a USA population under 40 years of age [14]. The age of diagnosis for ET cases in Calgary reported here has a relatively low median of 61 , however the highest incidence still occurs in the advanced age group of 80-84 years. In light of the sex and age biases MPN incidence, it would be interesting to examine the relationship between age of diagnosis and sex ratio in future studies. 
The calculated Canadian incidence rates for the MPN subtypes were near the range of published values for other regions (Table 2) except for PMF which was markedly high at 0.80 cases per 100,000 person-years. The corresponding crude incidence was 0.56 cases per 100,000 person-years. The elevated age-standardized rate may be largely attributed to Calgary having a relatively younger population and diagnoses occurring at advanced age, with 29 of the 38 PMF cases in adults 65 years or older. Relative differences in incidence of the MPNs between countries are partially due to inherent genetic and environmental factors, however variation in healthcare systems, diagnostic practice and reporting should also be taken in mind.

Unclassifiable MPN is defined as a heterogenous mix of incomplete diagnoses that cannot be differentiated into one of the classic subtypes, usually because the disease is in early stage. Here, MPN-U accounted for 17 cases of $139 \mathrm{MPN}$ (12.2\%), which falls within the estimated range of $10-15 \%$ [15]. The proportion of MPN-U's is expected to decrease as differential diagnostic criteria are undergoing refinement, especially in light of the updated 2017 WHO diagnostic criteria which are primarily focused on differentiating the MPN subtypes [16]. In a retroactive reclassification study using the 2016 criteria, the size of the MPN-U category was reduced by about $30 \%$ [17].

In the 5 years study period, three cases of MPN were observed in patients under 20 years old, the youngest at 8 years old. Early-onset disease is typically suggestive of genetic predisposition, however familial cases of MPN are very rare and the epidemiology of early-onset MPN is mostly unknown [18].

In summary we report for the first time a cumulative MPN incidence of 2.05 cases per 100,000 person-years in Calgary and an age-standardized rate of 2.71 cases per 100,000 person-years for Canada. This is in general agreement with literature values, however a high rate of primary myelofibrosis was observed, especially in males. Future studies should be conducted to further refine these estimates and build upon the epidemiology of the MPNs.

\section{Limitations}

Our reported rates were limited by the geography and study period, which occurred prior to the WHO revision to MPN subclassification. Furthermore, this study does not include additional epidemiology measures such as prevalence and survival rate.

\section{Abbreviations}

CML: chronic myeloid leukemia; ET: essential thrombocythemia; JAK2: Janus kinase 2; MPN: myeloproliferative neoplasm; MPN-U: unclassified MPN;
PMF: primary myelofibrosis; PV: polycythemia vera; WHO: World Health Organization.

\section{Acknowledgements}

The authors thank Marcus Vaska for assistance in literature searching.

\section{Authors' contributions}

$\mathrm{JH}$ analyzed the data and wrote the manuscript draft. MG and LN analyzed the data. CN and FRK conceived of the study, extracted the data and gave feedback on the manuscript. All authors read and approved the final manuscript.

\section{Funding}

Support for data collection and analysis was provided by a Canadian Institutes of Health Research Foundation Scheme Grant to CN [RN254781-333204]. The funder did not have a say in the study design or outcome.

\section{Availability of data and materials}

The datasets used and/or analyzed during this study are available from the corresponding author on reasonable request.

\section{Ethics approval and consent to participate}

Ethics approval for this study was obtained from the Health Research Ethics Board of Alberta Cancer Committee (Ethics ID HREBA.CC-16-0830). As approved by the ethics committee, consent for participation was waived. No individual patient data were reported here.

\section{Consent for publication}

Not applicable.

\section{Competing interests}

The authors declare that they have no competing interests.

\section{Author details \\ ${ }^{1}$ College of Medicine, University of Saskatchewan, Saskatoon, SK, Canada. 2 Department of Pathology and Laboratory Medicine, Cumming School of Medicine, University of Calgary, Calgary, AB, Canada. ${ }^{3}$ Alberta Public Laboratories (formerly Calgary Laboratory Services), Calgary, AB, Canada. ${ }^{4}$ Departments of Family Medicine and Community Health, Cumming School of Medicine, University of Calgary, Calgary, AB, Canada. ${ }^{5}$ Department of Medi- cal Genetics, Cumming School of Medicine, University of Calgary, Calgary, AB, Canada.}

Received: 18 January 2019 Accepted: 18 May 2019

Published online: 24 May 2019

\section{References}

1. Swerdlow SHC, Campo E, Harris NL, Jaffe ES, Pileri SA, Stein H, et al. WHO classification of tumours of haematopoietic and lymphoid tissues. 4 th ed. Lyon: IARC Press; 2008.

2. Tefferi A, Pardanani A. Myeloproliferative neoplasms: a contemporary review. JAMA Oncol. 2015:1:97-105.

3. Titmarsh GJ, Duncombe AS, McMullin MF, O'Rorke M, Mesa R, De Vocht F, et al. How common are myeloproliferative neoplasms? A systematic review and meta-analysis. Am J Hematol. 2014;89:581-7.

4. Deadmond MA, Smith-Gagen JA. Changing incidence of myeloproliferative neoplasms: trends and subgroup risk profiles in the USA, 1973-2011. J Cancer Res Clin Oncol. 2015;141:2131-8.

5. Roaldsnes C, Holst R, Frederiksen H, Ghanima W. Myeloproliferative neoplasms: trends in incidence, prevalence and survival in Norway. Eur J Haematol. 2017:98:85-93.

6. Moulard O, Mehta J, Fryzek J, Olivares R, lqbal U, Mesa RA. Epidemiology of myelofibrosis, essential thrombocythemia, and polycythemia vera in the European Union. Eur J Haematol. 2014;92:289-97.

7. Byun JM, Kim YJ, Youk T, Yang JJ, Yoo J, Park TS. Real world epidemiology of myeloproliferative neoplasms: a population based study in Korea 2004-2013. Ann Hematol. 2017:96:373-81.

8. Nguyen LT, Guo M, Naugler C, Rashid-Kolvear F. Incidence of chronic myeloid leukemia in Calgary, Alberta, Canada. BMC Res Notes. 2018;11:780. 
9. Tefferi A, Thiele J, Vardiman JW. The 2008 World Health Organization classification system for myeloproliferative neoplasms: order out of chaos. Cancer. 2009;115:3842-7.

10. Boniol M, Heanue M. Age-standardisation and denominators. In: Curado MP, Edwards B, Shin HR, Storm H, Ferlay J, Heanue M, editors. Cancer incidence in five continents, IARC scientific publication no 160, vol IX. 2008/01/01 edition. France: International Agency for Research on Cancer World Health Organization; 2008. p. 9-101.

11. Table 051-0056. Estimates of population by census metropolitan area, sex and age group for July 1, based on the Standard Geographical Classification (SGC) 2011, annual (persons). Ottawa. 2015. http://www5.statcan.gc. $\mathrm{ca} / \mathrm{cansim} / \mathrm{a} 26$ ?lang=eng\&id=0510056. Accessed $31 \mathrm{Jan} 2017$.

12. Table 051-0001. Estimates of population, by age group and sex for July 1, Canada, provinces and territories, annual (persons unless otherwise noted). Ottawa. 2015. http://www5.statcan.gc.ca/cansim/ a26?lang=eng\&id=510001. Accessed 31 Jan 2017.

13. Wilson EB. Probable inference, the law of succession, and statistical inference. J Am Stat Assoc. 1927;22:209-12.

14. Szuber N, Vallapureddy RR, Penna D, Lasho TL, Finke C, Hanson CA, et al. Myeloproliferative neoplasms in the young: Mayo Clinic experience with 361 patients age 40 years or younger. Am J Hematol. 2018;93:1474-84.
15. Thiele J, Kvasnicka HM, Vardiman J. Bone marrow histopathology in the diagnosis of chronic myeloproliferative disorders: a forgotten pearl. Best Pract Res Clin Haematol. 2006:19:413-37.

16. Swerdlow SHC, Campo E, Harris NL, Jaffe ES, Pileri SA, Stein H, et al. WHO classification of tumours of haematopoietic and lymphoid tissues. 4 th ed. Lyon: IARC Press; 2017.

17. Iurlo A, Gianelli U, Cattaneo D, Thiele J, Orazi A. Impact of the 2016 revised WHO criteria for myeloproliferative neoplasms, unclassifiable: comparison with the 2008 version. Am J Hematol. 2017;92:E48-51.

18. Rumi E, Cazzola M. Diagnosis, risk stratification, and response evaluation in classical myeloproliferative neoplasms. Blood. 2017;129:680-92.

\section{Publisher's Note}

Springer Nature remains neutral with regard to jurisdictional claims in published maps and institutional affiliations.
Ready to submit your research? Choose BMC and benefit from:

- fast, convenient online submission

- thorough peer review by experienced researchers in your field

- rapid publication on acceptance

- support for research data, including large and complex data types

- gold Open Access which fosters wider collaboration and increased citations

- maximum visibility for your research: over $100 \mathrm{M}$ website views per year

At BMC, research is always in progress.

Learn more biomedcentral.com/submissions 